\title{
Propagation of 2D Pressure Pulses in Lipid Monolayers and Its Possible Implications for Biology
}

\author{
J. Griesbauer, ${ }^{1,2}$ S. Bössinger, ${ }^{1,2}$ A. Wixforth, ${ }^{1}$ and M. F. Schneider ${ }^{2}$ \\ ${ }^{1}$ University of Augsburg, Experimental Physics I, D-86159 Augsburg, Germany \\ ${ }^{2}$ Department of Mechanical Engineering, Boston University, Boston, Massachusetts 02215, USA
}

(Received 27 June 2011; published 9 May 2012)

\begin{abstract}
The existence and propagation of acoustic pressure pulses on lipid monolayers at the air-water interface are directly observed by simple mechanical detection. The pulses are excited by small amounts of solvents added to the monolayer. Controlling the state of the lipid interface, we show that the pulses propagate at velocities $c$ following the lateral compressibility $\kappa$. This is manifested by a pronounced minimum in $c$ $(\sim 0.3 \mathrm{~m} / \mathrm{s})$ within the transition regime. The role of interface density pulses in biology is discussed, in particular, in the context of communicating localized alterations in protein function (signaling) and nerve pulse propagation.
\end{abstract}

DOI: 10.1103/PhysRevLett.108.198103

PACS numbers: 87.14.Cc, 87.90.+y

Hydrated interfaces are ubiquitous in biology and appear on all scales, from single ions or proteins to the network of the cell cytoskeleton or macroscopic lipid membrane assemblies, as, for instance, in our nervous system. Thermodynamically, these interfaces are characterized by their diagrams of state, which, in the case of lipid membranes, can be experimentally controlled using, for instance, the Langmuir technique.

Mechanical, electrical, and optical properties, as well as transitions of first and second order, have been studied thoroughly for a variety of different membrane types $[1,2]$. Furthermore, it was also demonstrated that channel-like transmembrane current fluctuations in pure lipid membranes and membranes from protein-lipid mixtures can be readily described by the membrane's equilibrium fluctuations [3-8].

While static properties of membranes, including phase transitions of first and second order, have been studied intensively $[1,2,9]$, there is only a little literature on nonequilibrium experiments. Some experiments using harmonically oscillating barriers [10-14], capillary waves on water $[15,16]$, or optically excited molecules [17] were mainly used to analyze the complex dynamical compressibility $\kappa$ of different surfactants on water. Apart from this, high-frequency electrical excitation of wave phenomena on lipid monolayers has also recently been examined using a set of planar electrodes [18]. However, considering the abundance of (lipid) interfaces in biology, the lack of dynamic studies is surprising, as one would draw an important conclusion from the existence of, for instance, propagating pulses. It would, on the one hand, add facts to the debate on nerve pulse propagation, where the simultaneous manifestation of the action potential as a thermal, mechanical, optical, and also electrical pulse $[19,20]$ of reversible heat has been proposed to be explained in an analogy of propagating sound waves or more recently solitons [21-23], hence challenging the foundation of neurophysiology [19,24]. On the other hand, it would impose the question of how pulses propagating along interfaces regulate the function of the interface and incorporated or adsorbed proteins, in particular, enzymes and receptors. It has been shown, for example, that a change in the thermodynamic state of the membrane can regulate the activity of enzymes and pores [25-30]. A discussion of how such equilibrium observations combined with studies about dynamic propagation in those interfaces create a new, physical perspective on communication and cellular signaling in biology will be resumed at the end of this Letter.

From a thermodynamic perspective, pulse velocities should depend on the state (e.g., the compressibility) of the interface, which is here controlled using the Langmuir technique. For the latter, monomolecular films (monolayers) are spread on water, where they can be easily manipulated, for instance, by changing their lateral extension $A$ and monitoring their lateral pressure $\pi$. This lateral pressure is related to the isothermal compressibility $\kappa_{T}$ that is directly proportional to the negative inverse of the first derivative of the measured slope of $\frac{\partial \pi}{\partial A}$ at constant temperature.

Here, we follow this interesting idea and study the propagation of acoustic pulses within and along a lipid monolayer interface by directly observing the mechanical response of the excitable medium. In particular, our experiments prove the existence of propagating pressure pulse waves with amplitudes up to $0.3 \mathrm{mN} / \mathrm{m}$. We demonstrate that the measured propagation velocities are controlled by the thermodynamic state of the monolayer reflecting the same nonlinearity $c(\pi)$ as the compressibility $\kappa(\pi)$ in the phase transition regime. We present a simple linear hydrodynamic model which quantitatively describes our experimental findings surprisingly well.

Lipid monolayers of 1,2-dipalmitoyl-sn-glycero-3phosphocholine (DPPC) are spread from chloroform to the air-water interface of a film balance trough $[18,31]$ [Fig. 1(a)]. After 10 minutes of evaporation, the lateral pressure-area isotherm $(\pi-A)$ is recorded by slowly 

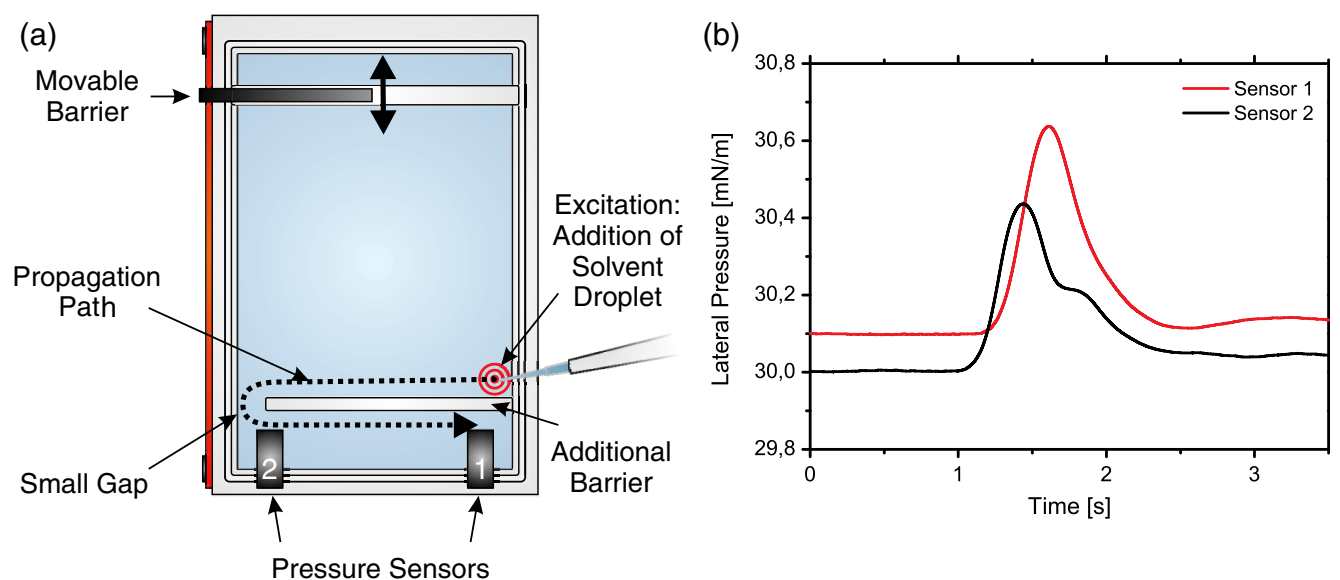

FIG. 1 (color online). (a) Film balance used for monolayer-pulse analysis. The trough is equipped with two pressure sensors and an additional barrier, which separates the excitation site from the detection compartment. (b) Time course of the sensor readouts for a pulse traveling from sensor 2 towards sensor 1 on a DPPC monolayer $\left(30 \mathrm{mN} / \mathrm{m}, 24^{\circ} \mathrm{C}\right)$. The time $t=0$ was arbitrarily chosen to be $1 \mathrm{~s}$ before the first pressure rise in the response of sensor 2. Before the pulse signal arrived at sensor 2, it had to propagate through the small gap between the additional barrier and the trough walls. To assure that the pressure pulse indeed travels the anticipated path, the additional barrier has been moved to the left side of the trough, opening the small gap near the excitation point (right). In fact, the order in which the pressure sensors responded was exactly reversed.

$\left[\sim 2.5 \AA^{2} /(\min \cdot\right.$ molecule $\left.)\right]$ compressing the film, employing a moveable barrier. The trough is equipped with two pressure sensors (Whilhelmy plates), which can be read out very rapidly $(10000$ samples $/ \mathrm{sec}, 0.01 \mathrm{mN} / \mathrm{m}$ resolution). These pressure sensors are used to directly and mechanically read out the response of arriving pressure pulses. The high sample rates allow for time-resolved measurement of the response and for Fourier transformation of the wave form. In order to exclude any spurious effects of water waves during the measurement of the twodimensional longitudinal pressure waves in the monolayer, an additional barrier is introduced in the trough [Fig. 1(a)]. The compression pulses in the lipid layer are excited in a separate compartment by a sudden addition of a small amount of solvent $(\sim 3 \mu \mathrm{l})$ to the monolayer surface (ethanol, methanol, chloroform, pentane). Because of the additional barrier, only waves which are able to travel over macroscopic distances and to pass the small gap will cause the two pressure sensors to respond. To exclude the possibility of water waves affecting the sensors, reference measurements with the solvents added to pure water surfaces were also recorded [32]. These measurements indeed do not show any recognizable signals.

In Fig. 1(b), a typical result of a propagating pulse following an excitation is shown. The pulse was excited at $t \sim 1 \mathrm{sec}$ by an ethanol droplet and the procedure described above. Shortly after the excitation, a well-defined pressure signal arrives at the Wilhelmy sensors at slightly different times. The time delay between the two signal sensors and their given separation in space $(\sim 14.5 \mathrm{~cm})$ then allows us to directly calculate the propagation velocity of the pressure pulse within the sensor compartment. To assure that the pressure pulse indeed travels the anticipated path, the additional barrier can also be moved to the other side of the trough, opening the small gap near the excitation point (right). As anticipated, then the order in which the pressure sensors respond is exactly reversed. Varying the path length by changing the position of excitation does not produce any resolvable differences in the measured pulse heights. This finding implies no or only very small attenuation of the pulses over the scale of the used trough $(20 \mathrm{~cm} \times 30 \mathrm{~cm})$. Indeed, our theoretical description (see below) as well as the preceding experiments ([12-14]) suggest attenuation constants of $\sim 1 \mathrm{~m}^{-1}$ and a wavelength of $\sim 1 \mathrm{~m}$. This would in turn render any possible attenuation and diffraction effects as basically immeasurable over the subjected propagation path $(\sim 20 \mathrm{~cm})$.

In Fig. 2, the temporal response of the Wilhelmy plate for four different excitation solvents in the liquid expanded phase of the monolayer is shown. Surprisingly, the pulse shapes appear not to be an inherent property of the film but to depend on the exciting solvent. While pulses excited from pentane and chloroform exhibit long tails $(\sim 20 \mathrm{~s})$, pulses of ethanol and methanol seem to produce rather sharp pulses. This well-reproducable observation might reflect the different residence times of the solvents in the monolayer, which in turn depend on the different water solubilities of the solvents. If the different pulses are Fourier-analyzed, all four spectra, however, reveal that the highest observable frequencies $(\sim 1 \mathrm{~Hz})$ depend only very little on the solvent.

We have recently shown ([18]) that high-frequency electrical stimulation of a lipid film also leads to wave phenomena with velocities around $100 \mathrm{~m} / \mathrm{s}$, as predicted by

$$
c_{0}=\sqrt{\frac{1}{\rho_{0} \kappa_{S}}}
$$




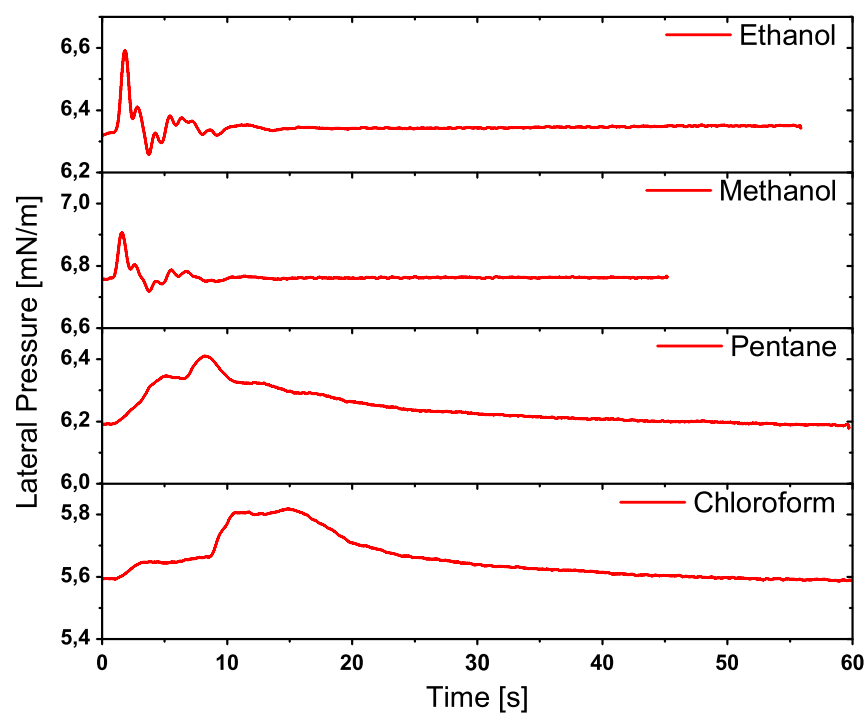

FIG. 2 (color online). Response of pressure sensor 1 for the excitation with four different solvents in the liquid expanded phase of a DPPC monolayer $\left(T=24^{\circ} \mathrm{C}\right)$. Again, the time $t=0$ was arbitrary chosen to be $1 \mathrm{~s}$ before the first pressure rise in the responses of sensor 2. Obviously, the measured pulse shapes depend on the solvent, whereas oscillations may be attributed to reflections of the wave within the propagation compartment (e.g., for ethanol). Pentane and chloroform, for example, remain longer in the monolayer $(\tau \sim 10 \mathrm{~s})$ before complete evaporation. Correspondingly, the recorded pulse extends over a longer period of time. A fast Fourier transform frequency analysis of the pulses suggests dominant frequencies up to $\omega_{\max } \sim 1 \mathrm{~Hz}$.

where $\rho_{0}$ is the film surface density and $\kappa_{S}$ is approximated by the isothermal lateral compressibility $\kappa_{T}$ directly extracted from a measured $\pi$-A isotherm. In contrast to these earlier results and to Eq. (1), our findings in this Letter as well as those of earlier studies [10,12-15,17] reveal that the observed propagation velocity of the pulses in the lipid film is rather slow $(\sim 1 \mathrm{~m} / \mathrm{s})$. One simple way to resolve the puzzle would be to account for viscous effects.

In this respect, the viscous force on a periodically $\left(\nu_{m}=\right.$ $A e^{i(\omega t-k x)}$ ) excited lipid monolayer coupled to a water subphase is given by [12]

$$
f \approx e^{i(\pi / 4)} \sqrt{\eta_{w} \rho_{w} \omega} v_{m} .
$$

Here, $\rho_{w}$ and $\eta_{w}$ denote the density and viscosity of water $[12,33]$. Neglecting transversal components and assuming $\kappa_{S}$ to be frequency-independent, we can introduce the viscous effects as an additional force into the Euler equation: $\rho_{0} \frac{\partial v_{m}}{\partial t}=\frac{-\partial \prod}{\partial x}-e^{i(\pi / 4)} \sqrt{\eta_{w} \omega \rho_{w}} v_{m}$. Using the continuity equation, one arrives at a wave equation for a monolayer being viscously coupled to the water underneath [32]

$$
\frac{\partial^{2} v_{m}}{\partial t^{2}}+\frac{1}{\rho_{0}} e^{i(\pi / 4)} \sqrt{\eta_{w} \rho_{w} \omega} \frac{\partial v_{m}}{\partial t}-c_{0}^{2} \frac{\partial^{2} v_{m}}{\partial x^{2}}=0 .
$$

Using the ansatz $v_{m}=A e^{i(\omega t-k x)}$, the dispersion relation for $\omega(k)$ can be extracted. For low frequencies $\omega$, the resulting attenuation $\beta$ and propagation velocity $c$ then turn out to be given by

$$
\begin{aligned}
& c=\frac{\omega}{\mathcal{R}(k)}=\cos ^{-1}\left(\frac{\pi}{8}\right) \sqrt{\frac{1}{\kappa_{S}} \sqrt{\frac{\omega}{\eta_{w} \rho_{w}}}}, \\
& \beta=-\Im(k)=\sin \left(\frac{\pi}{8}\right) \sqrt{\kappa_{S} \sqrt{\eta_{w} \rho_{w} \omega^{3}}} .
\end{aligned}
$$

As indicated by Eq. (4), the velocity $c$ is reduced with respect to $c_{0}=\sqrt{1 / \rho_{0} \kappa}$ (1) by a factor of the order of $\cos ^{-1}\left(\frac{\pi}{8}\right) \sqrt{\rho_{0} \sqrt{\omega / \eta_{w} \pi_{w}}}$, which, for $\omega \sim 1 \mathrm{~Hz}$, is $\sim 10^{-3}$. Since only insoluble lipid monolayers (DPPC) were investigated, further details like the diffusion of lipids into the subphase have not been treated here but have been discussed elsewhere in the context of capillary wave theory $[10,11]$.

In Fig. 3, we compare our experimental results to Eq. (4) for all four solvents investigated. The lines with squares represent the directly measured velocities, whereas the lines without squares have been calculated using $\omega \sim$ $1 \mathrm{~Hz}$ from the measured pulse form (Fig. 2) and the independently evaluated isothermal compressibility $\kappa_{T}=$ $\frac{-1}{A}\left(\frac{\partial A}{\partial \pi}\right)_{T}$. The velocities are plotted as a function of the applied lateral pressure $\pi$. As can be seen, we find very

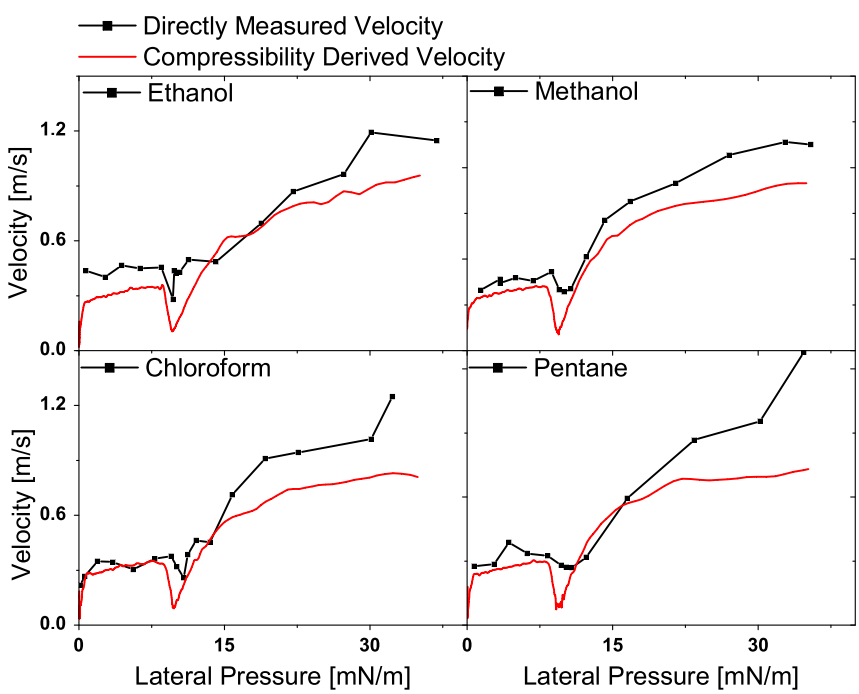

FIG. 3 (color online). Propagation velocities of the pulses, determined by the run time differences between sensors 2 and 1 (distance $d_{21} \sim 14.5 \mathrm{~cm}$ ) for different lateral pressures of the DPPC monolayer $\left(T=24^{\circ} \mathrm{C}\right)$. Additionally, the model according to Eq. (4) and the isothermal compressibility $\kappa_{T}$ are plotted into the corresponding graphs for an average pulse frequency of $\langle\omega\rangle \sim 1 \mathrm{~Hz}$. Both curves coincide very well; even the typical $\left(\kappa_{T}\right)^{-1}$ minima in the phase transition regime of the DPPC monolayer $\left(24^{\circ} \mathrm{C}\right)$ are well-reproduced. 
good agreement for the entire range of compressibilities and for all four solvents. Both the linear and nonlinear (region of maximum in $\kappa$ ) regimes including the characteristic dip in the velocity profile at the maximum of the compressibility are clearly resolved in the model and the experiment. The velocity decreases from $c=1.5 \mathrm{~m} / \mathrm{s}$ in the liquid condensed phase to $c=0.4 \mathrm{~m} / \mathrm{s}$ in the liquid expanded phase with a minimum of $c=0.2 \mathrm{~m} / \mathrm{s}$ in the transition regime, demonstrating the close relation between the thermodynamic state and the propagation velocity.

For the evaluations above, the assumption was made that $\kappa_{T} \approx \kappa_{S}$, which is probably oversimplified and may explain the small differences of measurement and theoretical expectations in Fig. 2. Equations (4) and (5) are only exact if the adiabatic compressibility $\kappa_{S}$ is used. Hence, the real dynamic or adiabatic compressibility $\kappa_{S}$ as a function of area or lateral pressure may be extracted using Eq. (4) and measurements of the propagation velocity. Comparing this $\kappa_{S}$ to $\kappa_{T}$, we find that, according to thermodynamic expectations [33], $\kappa_{S}<\kappa_{T}$ over the entire pressure spectrum. As indicated before $([11,34])$, the maximum of $\kappa_{S}$ is less pronounced than that of $\kappa_{T}$ [32].

Finally, we studied the degree of excitability of the interface in the different thermodynamic states. Therefore, the detected peak height of the propagating pressure pulse was analyzed for different lateral pressures and is plotted in Fig. 4. Close to the maximum of the compressibility, the detected pulse signal becomes very weak and is barely detectable. We believe that this directly proves that the thermodynamic state of the interface being controlled by the lipid monolayer not only influences the propagation speed but also its "strength."

In summary, we have given direct experimental proof that longitudinal pulses in lipid monolayers can be

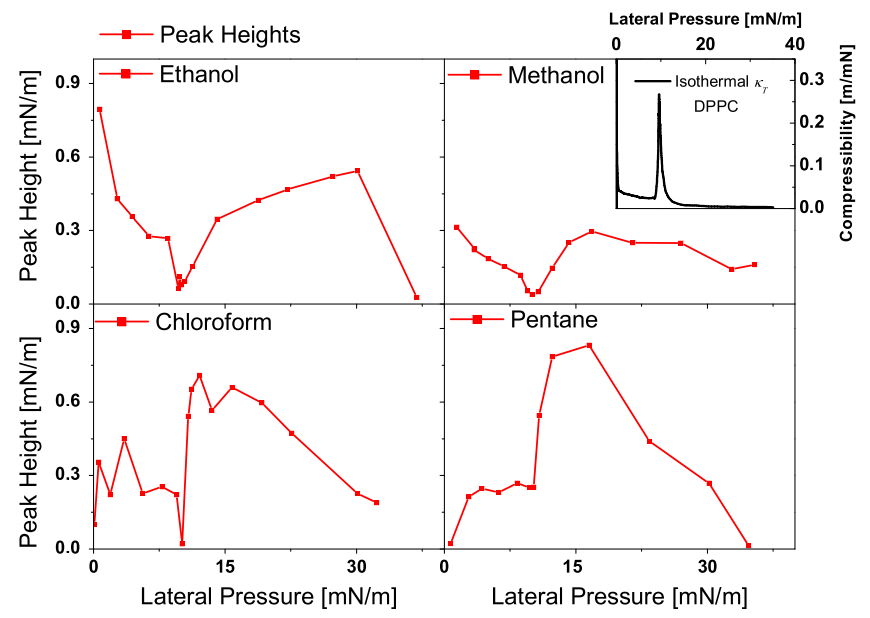

FIG. 4 (color online). Measured pulse heights as a function of lateral pressure in the lipid film (DPPC; $T=24^{\circ} \mathrm{C}$ ). Similar to the propagation velocity, a distinct minimum evolves at the compressibility maximum, indicating the phase transition regime. Hence, both pulse velocities and heights depend on the phase state of the DPPC monolayer. mechanically/chemically excited and depend on the thermodynamic state of the interface.

Realizing the general thermodynamic foundation of the phenomena and the ubiquity of soft interfaces in biological systems, our findings may have far-reaching consequences: On the one hand, it adds to the discussion about nerve pulse propagation as mentioned above in the sense that it demonstrates the ability of $2 \mathrm{D}$ density waves to propagate indeed over macroscopic distances $[22,35,36]$. On the other hand, it allows us to propose the new idea of communicating localized events crucial for the understanding of intra- and intercellular signaling in biology by combining our results with early, equilibrium studies that have demonstrated that membrane proteins can be regulated by the thermodynamic state of the interface [26-30]. Consider that a localized perturbation $p$ at one end (say at $X$ ) of an interface-induced, for instance, by a conformational change of a protein or the adsorption of a molecule, etc.- - triggers a propagating density pulse which changes the state of the interface [37] as it travels along. An enzyme or protein located at $Y$ will experience the collective changes of the arriving density pulse in its surroundings, which will modulate its activity [38] and possibly induce (or suppress) a new event $p^{\prime}$ that in turn triggers the propagation of a second pulse, and so on. This way, the localized events $p$ and $p^{\prime}$ (e.g., chemical reactions) at $X$ and $Y$ communicate through nonlocalized pulses along the integrating continuous interface. Future experiments on proteins incorporated into lipid membranes will have to test the relevance of this new hypothesis of nonlocalized coupling.

To analyze these ideas quantitatively, far more thorough theories will be necessary. At least three fundamental aspects need to be addressed: (i) the viscoelastic appearance of the interface itself (which has been neglected here) and the corresponding dispersion relation; (ii) the nonlinear response contributions, in particular, near maximum compressibility; and (iii) a complete thermodynamic description of nonequilibrium and equilibrium properties including the coupling of all the observables (area, charge, $p \mathrm{H}$, energy, etc.), including those between the interface state and the protein action. It will be interesting to explore how much of biology can be explained by the concepts of thermodynamics applied to the integrating interface.

M.F.S. thanks Dr. K. Kaufmann (Göttingen) who inspired him to work in this field and introduced him to the thermodynamic origin of the phenomena and Einstein's approach to statistical physics. We would like to also thank Dr. K. Kaufmann for his numerous seminars and discussions. Financial support by BU and the BMBF is gratefully acknowledged. M.F.S. thanks the Bavarian Science Foundation for financial support. This work has also been partially funded by the German Excellence Initiative "Nanosystems Initiative Munich, NIM" and the 
Deutsche Forschungsgemeinschaft, DFG (SPP1313 and WI1091/12-1).

[1] O. Albrecht, H. Gruler, and E. Sackmann, J. Phys. (Paris) 39, 301 (1978).

[2] G. L. Gaines, Insoluble Monolayers at Liquid-Gas Interfaces (Wiley, New York, 1966).

[3] A. Blicher, K. Wodzinska, M. Fidorra, M. Winterhalter, and T. Heimburg, Biophys. J. 96, 4581 (2009).

[4] K. Kaufmann, W. Hanke, and A. Corcia, Ion Channel Fluctuations in Pure Lipid Bilayer Membranes: Control by Voltage (Interscience, New York, 1989).

[5] J. F. Nagle and H. L. Scott, Biochim. Biophys. Acta 513, 236 (1978).

[6] B. Wunderlich, C. Leirer, A.-L. Idzko, U. F. Keyser, A. Wixforth, V. M. Myles, T. Heimburg, and M. F. Schneider, Biophys. J. 96, 4592 (2009).

[7] V.F. Antonov, V. V. Petrov, A. A. Molnar, D. A. Predvoditelev, and A.S. Ivanov, Nature (London) 283, 585 (1980).

[8] V.F. Antonov, A. A. Anosov, V.P. Norik, and E. Y. Smirnova, Eur. Biophys. J. 34, 155 (2005).

[9] H. Möhwald, Structure and Dynamics of Membranes, edited by R. Lipowsky and E. Sackmann (Elsevier, New York, 1995).

[10] B. A. Noskov, D. A. Alexandrov, and R. Miller, J. Colloid Interface Sci. 219, 250 (1999).

[11] J. Lucassen and M. van den Tempel, Chem. Eng. Sci. 27, 1283 (1972).

[12] J. Lucassen and M. van den Tempel, J. Colloid Interface Sci. 41, 491 (1972).

[13] J. Lucassen, Trans. Faraday Soc. 64, 2221 (1968).

[14] J. Lucassen, Trans. Faraday Soc. 64, 2230 (1968).

[15] W. Budach and D. Möbius, Thin Solid Films 178, 61 (1989).

[16] V. Vogel and D. Möbius, Langmuir 5, 129 (1989).

[17] M. Y. Suzuki, D. Möbius, and R. Ahuja, Thin Solid Films 138, 151 (1986).

[18] J. Griesbauer, A. Wixforth, and M. F. Schneider, Biophys. J. 97, 2710 (2009).

[19] J. M. Ritchie and R. D. Keynes, Q. Rev. Biophys. 18, 451 (1985).
[20] I. Tasaki, Biochem. Biophys. Res. Commun. 215, 654 (1995).

[21] E. Wilke, Pflügers Arch. 144, 35 (1912).

[22] K. Kaufmann, Action Potentials (Caruaru, Brazil, 1989).

[23] T. Heimburg and A.D. Jackson, Proc. Natl. Acad. Sci. U.S.A. 102, 9790 (2005).

[24] A. L. Hodgkin and A. F. Huxley, Bull. Math. Biol. 52, 25 (1990).

[25] T. Honger, K. Jorgensen, R. L. Biltonen, and O. G. Mouritsen, Biochemistry 35, 9003 (1996).

[26] G. Lenaz, G. Curatola, L. Mazzanti, and G. ParentiCastelli, Mol. Cell. Biochem. 22, 3 (1978).

[27] J.A.F. Op den Kamp, M. T. Kaurez, and L.L. M. van Denen, Biochim. Biophys. Acta 406, 169 (1975).

[28] H. Sandermann, Biochim. Biophys. Acta 515, 209 (1978).

[29] R. Verger and G. H. de Haas, Annu. Rev. Biophys. Bioeng. 5, 77 (1976).

[30] H. M. Seeger, L. Aldrovandi, A. Alessandrini, and P. Facci, Biophys. J. 99, 3675 (2010).

[31] D. Steppich, J. Griesbauer, T. Frommelt, W. Appelt, A. Wixforth, and M.F. Schneider, Phys. Rev. E 81, 061123 (2010).

[32] See Supplemental Material at http://link.aps.org/ supplemental/10.1103/PhysRevLett.108.198103 for theoretical derivations of formulas used in this Letter; moreover, additional measurements concerning the calculable adiabatic compressibility, phase-dependent pulse shapes, and reference measurements on pure water surfaces are provided.

[33] L. D. Landau and E. M. Lifschitz, Lehrbuch Der Theoretischen Physik - VI - Hydrodynamik (AkademieVerlag, Harri Deutsch, 2007).

[34] B. A. Noskov and D. A. Alexandrov, Colloids Surf. A 156, 307 (1999).

[35] E. Wilke, Pflügers Arch. 144, 35 (1912).

[36] T. Heimburg and A.D. Jackson, Proc. Natl. Acad. Sci. U.S.A. 102, 9790 (2005).

[37] Mechanically, the state is characterized, for instance, by its $\pi$-A diagram of state, but electrically $U-q$, thermally $E-T$, or chemically $\mu-N$ may be more proper representations under different circumstances.

[38] This should hold at least when time scales of the protein (in an enzyme, for instance, its turnover rate) are shorter than the inverse frequency of the pulse. 